\title{
A tour of VHE emitting AGNs: prospects with CTA
}

\author{
J.-P. Lenain ${ }^{* a b c}$, M. K. Daniel ${ }^{b}$, C. Boisson ${ }^{c}$, H. Sol ${ }^{c}$, P. M. Chadwick ${ }^{b}$, M. J. Ward ${ }^{b}$ \\ ${ }^{a}$ ISDC Data Centre for Astrophysics, Center for Astroparticle Physics, Observatoire de Genève, \\ Université de Genève, Chemin d'Ecogia 16, 1290 Versoix, Switzerland \\ ${ }^{b}$ University of Durham, Department of Physics; South Road, Durham DH1 3LE, U.K. \\ ${ }^{c}$ LUTH, Observatoire de Paris, CNRS, Université Paris Diderot; 5 Place Jules Janssen, 92190 \\ Meudon, France \\ E-mail: jean-philippe.lenain@obspm.fr
}

\begin{abstract}
About 47 very high energy (VHE; $E>100 \mathrm{GeV}$ ) emitting active galactic nuclei (AGNs) are detected with the current generation of imaging atmospheric Čerenkov telescopes (IACTs) so far, the major part of which belonging to the blazar class. We interpret the spectral energy distributions (SEDs) of these objects as simple stationary synchrotron self-Compton emission, and study the common properties of the sources derived from the SED modelling. We find a significant correlation between the luminosity in the $\mathrm{X}$-ray range $(2-10 \mathrm{keV})$ and the monochromatic luminosity at $1 \mathrm{TeV}$ among the currently detected VHE AGNs. Applying this relationship to other sources using the BZCat catalogue of AGNs, we propose a list of about 70 new source candidates at VHE in the framework of future obseravtions with current IACTs, and especially with the future Čerenkov Telescope Array (CTA).
\end{abstract}

AGN Physics in the CTA Era - AGN2011,

May 16-17, 2011

Toulouse, France

* Speaker. 


\section{Introduction}

Among active galactic nuclei (AGNs), those possessing a jet closely aligned to the line of sight, the so-called blazars, form the major part of extragalactic objects detected so far at very high energies (VHE; $E>100 \mathrm{GeV}$ ) with imaging atmospheric Čerenkov telescopes (IACTs). We present a simple, one-zone, stationary modelling of the spectral energy distribution (SED) for the VHE emitting AGNs detected at VHE so far, for which we endeavoured to describe low states of activity, and discuss common properties among these objects. We propose a list of source candidates at VHE based on a correlation between X-ray and VHE luminosities that we found in our sample, following the modelled SEDs we present for the known VHE emitting AGNs.

\section{The model}

We will focus here on a leptonic description of the SED of VHE AGNs. The absorption of the VHE part of the energy spectrum by the extragalactic infrared background light is accounted for, using the prescription of $[1,2]$.

We use the so-called "blob-in-jet" description (see e.g. [3]), in which the high energy synchrotron self-Compton (SSC) emitting region is a dense, compact region filled by a tangled magnetic field, embedded in an extended inhomogeneous jet, where synchrotron radiation dominates the low frequency part - up to the optical range - of the energy spectrum. For all the retrieved data from the different VHE AGNs, we consistently apply the same model to their SED, keeping the Doppler factor around $\delta_{b} \sim 20$, the magnetic field strength $B \sim 0.1 \mathrm{G}$ and the first index $n_{1} \sim 2$ for the broken power-law describing the lepton energy distribution (LED) in the source, in order to reduce the number of free parameters in the model. The density of the relativistic particles, the size of the emitting region, the second slope and the energy break of the LED are let free to vary.

\section{The sample}

Currently, 47 extragalactic sources have been detected at VHE, the vast majority of which belonging to the class of BL Lac objects. We focus on sources with published VHE spectral data so far, which comes to 25 sources, and are all blazars, except the radio galaxies M 87 [4] and Cen A [5], and the quasar 3C 279 [6]. Since these objects are highly variable at all wavelengths, we endeavour to describe the lower state of activity for their SED known for each source with a stationary SSC model.

The purpose of this sample study is first to assess whether a simple SSC modelling can account for the SEDs of the considered sources, and then if correlations emerge amongst derived parameters from the model compared to the data. One shoud however note that the sample is observationally biased towards high-frequency-peaked BL Lac objects and flaring states, because of the observation strategy of current IACT experiments.

As an illustration, the multi-wavelength emission of the well studied high-frequency-peaked BL Lac object PKS 2155-304 is presented in Fig. 1, in which the lowest state of activity ever recorded during the 2003 multi-wavelength campaign is reported [7], the 2008 campaign including Fermi/LAT data during which the VHE flux is at the same level as in 2003 [8], and for comparison the second big VHE flare detected with H.E.S.S. in 2006 [9]. 

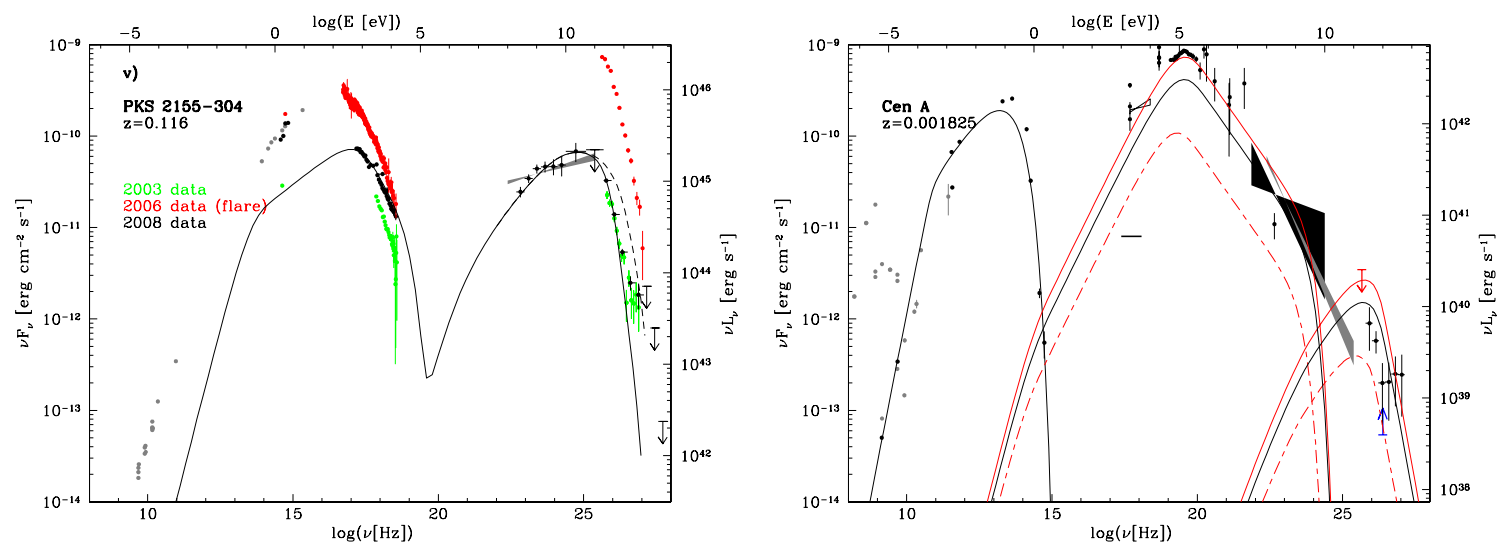

Figure 1: Left: SED of PKS 2155-304. The green data point represent the multi-wavelength data taken during a low state of activity in 2003 [7]. The black points at high energies show a more recent campaign making use of Fermi/LAT data [8]. For comparison, we show the big flare which occurred on July 28, 2006 [9]. The grey points are data from the extended jet, dominating the emission at low frequencies and which model is not presented here. Right: SED of Cen A. The black bow-tie at high energy are EGRET data, and the grey bow-tie is taken from the Fermi/LAT first source catalogue [10]. For more details on the model, see [11].

We show as well in Fig. 1 the SED of Cen A, the closest radio galaxy, eventually detected with H.E.S.S. after $115 \mathrm{~h}$ of good quality observations [5]. The VHE spectrum of this source was predicted in [11], and we add the VHE spectrum measured by H.E.S.S. to the predicted SED.

\section{Discussion}

A correlation between the integrated synchrotron and the integrated SSC emissivities is naturally expected in SSC models for a sample of sources. From the SED modelling of these currently detected VHE emitting AGNs conducted in this work, we find a significant correlation (correlation coefficient $\rho=0.85$, corresponding false alarm probability $p \sim 10^{-7}$ ) between observables in the sample, which are the modelled X-ray luminosity in the $2-10 \mathrm{keV}$ range and the modelled, monochromatic luminosity at $1 \mathrm{TeV}$ (see Fig. 2):

$$
\log _{10}\left(L_{1 \mathrm{TeV}}\left[\mathrm{erg} \mathrm{s}^{-1}\right]\right)=(0.993 \pm 0.003) \log _{10}\left(L_{\mathrm{X}}\left[\mathrm{erg} \mathrm{s}^{-1}\right]\right)
$$

We use X-ray fluxes given in the BZCat catalogue [12] to assess the expected flux in VHE $\gamma$ rays from this relationship, and put a lower cut on the expected VHE flux of $10^{-13} \mathrm{~cm}^{-2} \mathrm{~s}^{-1} \mathrm{TeV}^{-1}$, which is the expected sensitivity limit at $1 \mathrm{TeV}$ for a detection at $5 \sigma$ confidence level in $50 \mathrm{~h}$ of observations with the future Čerenkov Telescope Array (CTA). The BZCat catalogue groups together BL Lac objects (coded as BZB), FSRQ (as BZQ), and blazars of uncertain/transitional type (as BZU), the latter including sources which are not very radio-loud. However, our derivation of a list of VHE candidates from this catalogue relies on the modelling of mainly BL Lac, which are radioloud objects. Our predictions for the VHE candidates should thus be considered strongly safer for the BZB sources than for the other types BZQ and BZU. We thus propose to test the following hypothesis: could our method be extrapolated to radio-quiet objects? If, for instance, the accretion 


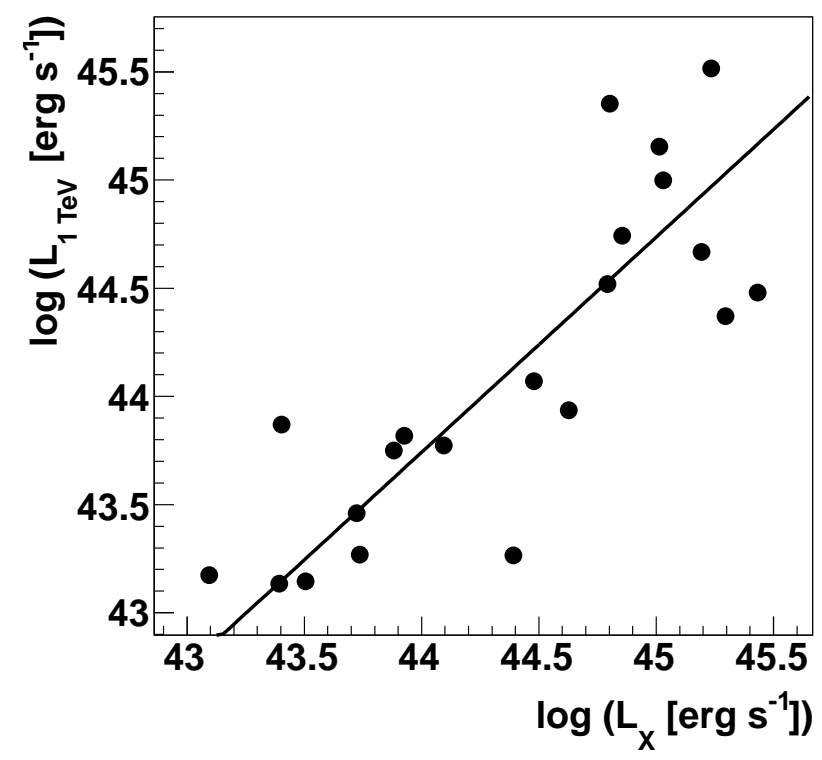

Figure 2: Correlation between the luminosities in the $\mathrm{X}$-ray range and at $1 \mathrm{TeV}$ for the currently detected VHE AGNs.

disc dominates the X-ray luminosity in some objects, the term $L_{X}$ in the relation above should be only a fraction, coming from the jet, of the total X-ray luminosity.

We end up with 71 VHE AGN candidates, the brightest of which should already be detectable with current IACTs, such as NGC 7213 or BZB J1728+5013. We report in Table 1 the 15 source candidates with the brightest expected VHE flux. It is noticeable that among the 21 objects used in [13] to derive a $4.9 \sigma$ detection of a stacked sample of AGNs with MAGIC, 5 sources are common to our candidates. One should note that our proposed method is conservative in the sense that it is based on the monochromatic luminosity at $1 \mathrm{TeV}$, while CTA will have an energy threshold as low as $\sim 30 \mathrm{GeV}$. This should increase the expected number of such sources, especially since the VHE spectra of blazars are usually steep.

With a better sensitivity and increased field of view compared to current IACTs, CTA is well suited to conduct a large population study on VHE AGNs, with the possibility of detecting objects further away than currently achieved ${ }^{1}$, as well as detecting many more fainter sources. Such a population study will allow one:

- to better constrain the flux level and spectral shape of the extragalactic background light $[14,15]$,

- to increase the statistics of detected sources ${ }^{2}$,

- to detect new type of sources ${ }^{3}$,

\footnotetext{
${ }^{1}$ The furthest known VHE object is the quasar 3C 279 [6] with a redshift of $z=0.536$.

${ }^{2}$ Less than 50 AGNs are currently known in VHE. More detections are particularly required for complex objects which jet is misaligned to the line of sight, such as radio galaxies and flat spectrum radio quasars.

${ }^{3}[16]$ interestingly reported the detection of the Seyfert 2 galaxy NGC 1068 at high energy using Fermi/LAT data.
} 


\begin{tabular}{lccccc}
\hline \hline Source denomination & $z$ & $F_{X}\left(\mathrm{erg} \mathrm{cm}^{-2} \mathrm{~s}^{-1}\right)$ & $L_{X}\left(\mathrm{erg} \mathrm{s}^{-1}\right)$ & $F_{1 \mathrm{TeV}}\left(\mathrm{erg} \mathrm{cm}^{-2} \mathrm{~s}^{-1}\right)$ & $L_{1 \mathrm{TeV}}\left(\mathrm{erg} \mathrm{s}^{-1}\right)$ \\
\hline BZU J0319+4130 & 0.018 & $2.0 \times 10^{-10}$ & $1.4 \times 10^{44}$ & $7.2 \times 10^{-11}$ & $7.0 \times 10^{43}$ \\
BZU J2209-4710 & 0.006 & $3.5 \times 10^{-11}$ & $2.8 \times 10^{42}$ & $1.6 \times 10^{-11}$ & $1.4 \times 10^{42}$ \\
BZU J0433+0521 & 0.033 & $2.3 \times 10^{-11}$ & $5.5 \times 10^{43}$ & $6.4 \times 10^{-12}$ & $2.7 \times 10^{43}$ \\
BZU J1407-2701 & 0.022 & $1.5 \times 10^{-11}$ & $1.6 \times 10^{43}$ & $5.3 \times 10^{-12}$ & $8.2 \times 10^{42}$ \\
BZB J1728+5013 & 0.055 & $2.0 \times 10^{-11}$ & $1.4 \times 10^{44}$ & $3.9 \times 10^{-12}$ & $6.8 \times 10^{43}$ \\
BZU J0425-0833 & 0.039 & $1.2 \times 10^{-11}$ & $4.1 \times 10^{43}$ & $3.1 \times 10^{-12}$ & $2.0 \times 10^{43}$ \\
BZU J1715+5724 & 0.027 & $7.4 \times 10^{-12}$ & $1.2 \times 10^{43}$ & $2.3 \times 10^{-12}$ & $6.0 \times 10^{42}$ \\
BZB J2123-1036 & 0.023 & $6.4 \times 10^{-12}$ & $7.5 \times 10^{42}$ & $2.2 \times 10^{-12}$ & $3.7 \times 10^{42}$ \\
BZU J1840-7709 & 0.018 & $5.9 \times 10^{-12}$ & $4.3 \times 10^{42}$ & $2.2 \times 10^{-12}$ & $2.1 \times 10^{42}$ \\
BZU J0522-3627 & 0.055 & $1.0 \times 10^{-11}$ & $7.1 \times 10^{43}$ & $2.0 \times 10^{-12}$ & $3.5 \times 10^{43}$ \\
BZQ J1229+0203 & 0.158 & $6.3 \times 10^{-11}$ & $3.7 \times 10^{45}$ & $1.5 \times 10^{-12}$ & $1.8 \times 10^{45}$ \\
BZB J1117+2014 & 0.139 & $3.4 \times 10^{-11}$ & $1.5 \times 10^{45}$ & $1.2 \times 10^{-12}$ & $7.3 \times 10^{44}$ \\
BZB J1217+3007 & 0.130 & $2.5 \times 10^{-11}$ & $9.8 \times 10^{44}$ & $1.1 \times 10^{-12}$ & $4.8 \times 10^{44}$ \\
BZB J0643+4214 & 0.089 & $1.1 \times 10^{-11}$ & $1.9 \times 10^{44}$ & $1.1 \times 10^{-12}$ & $9.4 \times 10^{43}$ \\
BZB J1443-3908 & 0.065 & $6.6 \times 10^{-12}$ & $6.3 \times 10^{43}$ & $1.0 \times 10^{-12}$ & $3.1 \times 10^{43}$ \\
$\vdots$ & $\vdots$ & $\vdots$ & $\vdots$ & $\vdots$ & $\vdots$ \\
\hline
\end{tabular}

Table 1: Prospects for future VHE AGN source candidates based on the BZCat catalogue. The sources are sorted by decreasing expected VHE flux. At $1 \mathrm{TeV}$, the luminosity is given as intrinsic to the source, corrected for the EBL, while the corresponding flux density is given as observed, absorbed by the EBL. Because of the unknown nature of BZU objects, the corresponding predictions should be taken with special care, and only apply under the assumption that the radiation is dominated by the jet emission. These cases are reported in italic.

- to derive the luminosity function of VHE AGNs and study the contribution of these AGNs to a potential extragalactic VHE $\gamma$-ray diffuse background (see also [17]).

Considering an array candidate well suited for the low energy part of spectrum for CTA, such as the array candidate E (see [18]), we expect the 16 brightest objects of our list of candidates to be detected in $50 \mathrm{~h}$ of total observations, using the CTA system in sub-array observation mode of 4 or 5 telescopes. For the remaining sources, we expect an average observation time of about $20 \mathrm{~h}$ per source to achieve a $5 \sigma$ detection with the full array. Assuming that $\sim 30 \%$ of the overall CTA observation time would be allocated to AGN observations, all the 71 proposed sources should be detected during an observational program of less than 2 years.

\section{Conclusion}

Currently detected VHE AGNs have been modelled using a simple, stationary SSC scenario, which describes well the broadband properties of the sources in low states of activity. From this sample, we found a correlation between the X-ray and $1 \mathrm{TeV}$ luminosities. We used the X-ray fluxes reported in the BZCat catalogue of AGNs to estimate their VHE flux and propose a list of new AGN candidates in the VHE range, in the prospects of future observations of IACTs, and particularly the future VHE observatory, CTA. The proposed observational strategy would allow to more than double the number of VHE AGNs in less than 2 years, for the expected sensitivity of CTA which will be by a factor of 10 better than current IACTs. 


\section{Acknowledgments}

J.-P. L. acknowledges receipt of a fellowship funded by the European Commission's Framework Programme 6, through the Marie Curie Early Stage Training project MEST-CT-2005-021074. J.-P. L. would like to warmly thank all the colleagues from the Astronomy group of the Department of Physics at Durham University, UK, for their welcome during his visit. We are grateful to a lot of people from the VHE astronomy community, too numerous to be quoted here, who kindly shared their VHE data.

This research made use of the SIMBAD database, operated at CDS, Strasbourg, France, and of the NASA/IPAC Extragalactic Database (NED), which is operated by the Jet Propulsion Laboratory, California Institute of Technology, under contract with the National Aeronautics and Space Administration. This work is partly based on multi-frequency archival data taken from the ASDC, a facility of ASI, and made use of data obtained from the High Energy Astrophysics Science Archive Research Center (HEASARC), provided by NASA's Goddard Space Flight Center, as well as of the TeVCat online catalogue for VHE $\gamma$-ray astronomy.

Finally, J.-P. L. would like to dedicate this work to the memory of his missed friend JeanClaude Rouffignat.

\section{References}

[1] T. M. Kneiske, K. Mannheim, \& D. H. Hartmann, A\&A, 386, 1, 2002 [astro-ph/ 0202104 ].

[2] T. M. Kneiske, T. Bretz, K. Mannheim, \& D. H. Hartmann, A\&A, 413, 807, 2004 [astro-ph/0309141].

[3] K. Katarzyński, H. Sol, \& A. Kus, $A \& A, \mathbf{3 6 7}$, 809, 2001.

[4] F. Aharonian, et al. (H.E.S.S. collaboration), Science, 314, 1424, 2006 [a st ro-ph/ 0612016 ].

[5] F. Aharonian, et al. (H.E.S.S. collaboration), ApJ, 695, L40, 2009 [0 903 . 1582].

[6] J. Albert, et al. (MAGIC collaboration), Science, 320, 1752, 2008 [arXiv: 0807 . 2822].

[7] F. Aharonian, et al. (H.E.S.S. collaboration), A\&A, 442, 895, 2005 [astro-ph/ 0506593 ].

[8] F. Aharonian, et al. (H.E.S.S. collaboration), ApJ, 696, L150, 2009 [arXiv: 0903.2924 ].

[9] F. Aharonian, et al. (H.E.S.S. collaboration), A\&A, 502, 749, 2009 [arXiv: 0906.2002 ].

[10] A. A. Abdo, et al. (Fermi/LAT collaboration), ApJS, 188, 405, 2010 [arXiv: 1002 . 2280].

[11] J.-P. Lenain, C. Boisson, H. Sol, \& K. Katarzyński, A\&A, 478, 111, 2008 [arXiv: 0710.2847 ].

[12] E. Massaro, P. Giommi, C. Leto, et al., A\&A, 495, 691, 2009 [arXiv: 0810.2206 ].

[13] J. Aleksić, et al. (MAGIC collaboration), ApJ, 729, 115, 2011 [arXiv: 1002 .2951].

[14] M. G. Hauser \& E. Dwek, ARA\&A, 39, 249, 2001 [astro-ph/0105539].

[15] F. Aharonian, et al. (H.E.S.S. collaboration), Nature, 440, 1018, 2006 [ast ro-ph/ 0508073 ].

[16] J.-P. Lenain, C. Ricci, M. Türler, D. Dorner, \& R. Walter, A\&A, 524, A72, 2010 [arXiv: 1008.5164$]$.

[17] Y. Inoue, T. Totani, \& M. Mori, PASJ, 62, 1005, 2010 [arXiv: 1002 . 4 782].

[18] The CTA Consortium, 2010 [arXiv: 1008 .3703]. 